\title{
PLK1 inhibition enhances temozolomide efficacy in IDH1 mutant gliomas
}

\author{
Robert F. Koncar ${ }^{1}$, Zhengtao Chu ${ }^{1}$, Lindsey E. Romick-Rosendale ${ }^{2}$, Susanne I. \\ Wells ${ }^{2}$, Timothy A. Chan ${ }^{3}$, Xiaoyang $\mathbf{Q i}^{1}$, El Mustapha Bahassi ${ }^{1}$ \\ ${ }^{1}$ Department of Internal Medicine, Division of Hematology/Oncology, University of Cincinnati, Cincinnati, OH, USA \\ ${ }^{2}$ Division of Oncology, Cincinnati Children's Hospital Medical Center, Cincinnati, OH, USA \\ ${ }^{3}$ Human Oncology and Pathogenesis Program, Memorial Sloan-Kettering Cancer Center, New York, NY, USA \\ Correspondence to: El Mustapha Bahassi, email: bhassiem@uc.edu
}

Keywords: glioma, IDH1, temozolomide, PLK1, checkpoint adaptation

Received: September 09, $2016 \quad$ Accepted: January 04, $2017 \quad$ Published: February 02, 2017

\section{ABSTRACT}

Despite multimodal therapy with radiation and the DNA alkylating agent temozolomide (TMZ), malignant gliomas remain incurable. Up to $90 \%$ of grades II-III gliomas contain a single mutant isocitrate dehydrogenase 1 (IDH1) allele. IDH1 mutant-mediated transformation is associated with TMZ resistance; however, there is no clinically available means of sensitizing IDH1 mutant tumors to TMZ. In this study we sought to identify a targetable mechanism of TMZ resistance in IDH1 mutant tumors to enhance TMZ efficacy. IDH1 mutant astrocytes rapidly bypassed the $\mathbf{G} 2$ checkpoint with unrepaired DNA damage following TMZ treatment. Checkpoint adaptation was accompanied by PLK1 activation and IDH1 mutant astrocytes were more sensitive to treatment with BI2536 and TMZ in combination ( $<20 \%$ clonogenic survival) than either TMZ ( $60 \%)$ or BI2536 ( 75\%) as single agents. In vivo, TMZ or BI2536 alone had little effect on tumor size. Combination treatment caused marked tumor shrinkage in all mice and complete tumor regression in 5 of 8 mice. Mutant IDH1 promotes checkpoint adaptation which can be exploited therapeutically with the combination of TMZ and a PLK1 inhibitor, indicating PLK1 inhibitors may be clinically valuable in the treatment of IDH1 mutant gliomas.

\section{INTRODUCTION}

Malignant gliomas are currently associated with a dismal prognosis and recurrence remains nearly inevitable despite a multimodal treatment strategy $[1,2]$. Gliomas are histologically graded as I-IV and $70-90 \%$ of grades II-III gliomas and secondary grade IV glioblastomas contain a mutation in one Isocitrate dehydrogenase 1 (IDH1) allele, with $\mathrm{R} 132 \mathrm{H}$ being the most common [3-5]. IDH1 is found in the cytoplasm and peroxisomes where it converts isocitrate to alpha ketoglutarate $(\alpha \mathrm{KG})$. However, the mutant enzyme converts $\alpha \mathrm{KG}$ into oncometabolite D-2hydroxyglutarate (D2HG), which is structurally similar to $\alpha \mathrm{KG}$ and a competitive inhibitor of $\alpha \mathrm{KG}$-dependent dioxygenases [6-8].

Treatment for gliomas typically consists of surgical resection, radiation therapy, and chemotherapy with the DNA alkylating agent, temozolomide (TMZ) [9]. The cytotoxic effect of TMZ is mediated primarily through generating O-6-methylguanine $(\mathrm{O} 6 \mathrm{meG})$ lesions [10]. If the methyl group is not removed by O-6-methylguanineDNA methyltransferase (MGMT), an enzyme associated with TMZ resistance, O6meG mispairs with thymine during DNA replication, leading to futile rounds of mismatch repair and persistent G2 checkpoint arrest followed by apoptosis or senescence [11]. MGMT promoter methylation and consequently low MGMT expression is typical in, but not unique to IDHI mutant gliomas [12], which generally respond better to TMZ than their IDHI wild type (WT) counterparts $[13,14]$. However, MGMT expression is not the sole determinant of TMZ sensitivity [15-18] and IDHI mutant and wildtype gliomas have different molecular ontogenies, making comparisons between IDHI mutant and wild type gliomas uninformative as to which tumor characteristics can be attributed directly to $I D H 1$ mutation. Grade II-III gliomas lacking the $I D H 1$ mutation are genetically distinct from IDH1 mutant gliomas and are more similar to primary 
grade IV glioblastomas. While genetic alterations such as EGFR amplification and $C D K N 2 A$ deletion are common in IDH1 WT gliomas, they rarely occur in gliomas with mutant $I D H 1$ [19]. Despite being considered chemoresponsive IDH1 mutant gliomas commonly recur even after surgical resection and treatment with radiation and temozolomide, highlighting the need for new treatment options [20-22].

Recent evidence suggests that IDHI mutantmediated transformation promotes TMZ resistance and rapid G2 checkpoint exit due to increased homologous recombination capability [23]. How IDH1 affects DNA repair and checkpoint signaling however, is unknown. The DNA damage checkpoint is a critical process that coordinates cell cycle progression with DNA damage repair. Thus, understanding how IDHI mutation affects checkpoint signaling may reveal ways to further sensitize IDH1 mutant tumor cells to TMZ.

Polo-like kinase 1 (PLK1) is a key regulator of mitotic progression following DNA damage-induced G2 checkpoint activation. It is involved in checkpoint recovery, which requires repair of damaged DNA, and checkpoint adaptation, in which cell division occurs with unrepaired DNA damage [24]. PLK1 is commonly overexpressed or over-activated in cancer, and is the target of several promising drugs in late stage clinical trials [25].

In this study, we sought to elucidate the mechanism of TMZ resistance and to identify potential targets to enhance TMZ efficacy in IDH1 mutant tumors. To this end, we used immortalized astrocytes to ask whether mutant IDH1 promotes TMZ resistance as a consequence of D2HG production and whether checkpoint adaptation, mediated through PLK1 activation rather than swift DNA damage repair accounts for the early progression out of G2 arrest. We show that IDH1 mutant cells and tumors can be greatly sensitized to TMZ by inhibiting PLK1 in vitro, as well as in a xenograft mouse model.

\section{RESULTS}

\section{IDH1 mutant-associated D2HG promotes TMZ resistance}

To study the effects of mutant IDH1, we used normal human astrocytes (NHA) which have been immortalized and described elsewhere [26]. When transformed by expression of an exogenous mutant IDH1 gene, the NHA epigenetically resemble IDH1 mutant gliomas [27]. A hemagglutinin (HA) tagged WT or $\mathrm{R} 132 \mathrm{H}$ mutant IDH1 gene was introduced into the NHA by retroviral transduction and gene expression was confirmed by Western blot (Figure 1A). WT and IDH1 $\mathrm{R} 13 \mathrm{H}$ clones showing comparable levels of exogenous wild type and mutant IDH1 proteins were selected. The WT and mutant cell lines were additionally confirmed by Sanger sequencing (Supplementary Figure 1A). NMR spectroscopy revealed increased $2 \mathrm{HG}$ concentrations in the IDH1 mutant cells (Supplementary Figure 1B).

After confirming the presence of the IDH1 mutation and $2 \mathrm{HG}$ production by the astrocytes we used them to test the effect of IDH1 mutation on TMZ sensitivity by clonogenic survival. After treatment with TMZ $(100 \mu \mathrm{M})$, mutant IDH1 NHA were significantly less sensitive to TMZ while WT NHA displayed an intermediate phenotype between the control and IDH1 mutant cells (Figure 1B), which is consistent with published data [23]. Differences in TMZ sensitivity were not due to differential MGMT expression as all three cell lines were MGMT deficient (Figure 1C).

The response of an MGMT deficient cell line such as NHA to TMZ is prolonged G2 arrest [11, 23]. This characteristic arrest was seen in the control NHA which displayed sustained G2 arrest up to 7 days after TMZ treatment (Figure 1D). However, IDH1 mutant NHA displayed a much shorter $\mathrm{G} 2$ arrest and by day 3 post TMZ treatment, the percentage of cells in G2 was similar to that of untreated cells (Figure 1D). IDH1 WT NHA again displayed an intermediate phenotype between the mutant and control NHA with sustained G2 arrest through Day 4 post TMZ.

Production of D2HG is considered to be the primary means by which mutant IDH1 promotes oncogenesis [7, $8,28]$. D2HG is required for maintenance of oncogenic properties of IDH1 mutant cells and has been shown to inhibit the activity of $\alpha \mathrm{KG}$-dependent dioxygenases which may play a role in cellular transformation $[6,7$, 28]. To determine if TMZ resistance conferred by IDH1 mutation is mediated by $\mathrm{D} 2 \mathrm{HG}$ production, we evaluated TMZ sensitivity in IDH1 mutant and parental NHA treated with D2HG, which has been shown to rapidly enter cells [29], at a concentration $(5 \mathrm{mM})$ within the range of what has been reported in IDH1 mutant gliomas $[6,30]$. In parental NHA, D2HG alone resulted in a mild but significant decrease in survival which is consistent with reported effects of D2HG treatment on glioma cell lines [29]. However, D2HG treatment resulted in increased survival of parental NHA treated with TMZ (Figure 1E), and there was a significant interaction between TMZ treatment and $\mathrm{D} 2 \mathrm{HG}$ treatment $(\mathrm{P}=0.02)$, indicating $\mathrm{D} 2 \mathrm{HG}$ production acutely promotes TMZ resistance. D2HG treatment had no effect on the IDH1 mutant astrocytes.

\section{IDH1 mutation promotes premature G2 checkpoint exit following TMZ treatment}

DNA damage-induced G2 checkpoint arrest is followed by checkpoint recovery, checkpoint adaptation, or apoptosis. While checkpoint recovery allows cell cycle progression after repair of damaged DNA, checkpoint adaptation allows mitotic progression despite unrepaired DNA damage [24]. To determine whether the shortened TMZ-induced G2 arrest in IDH1 mutant cells is due to 
efficient repair of damaged DNA or premature checkpoint override with residual unrepaired DNA damage, we measured total DNA damage by alkaline comet assay. IDH1 mutant astrocytes did not show statistically different levels of DNA damage than control and IDH1 WT astrocytes at days 1, 3, and 7 post TMZ (Figure 2A, 2B). We next specifically examined the more lethal double-strand breaks by neutral comet assay, and again found no statistically significant differences between the three cell types (Figure 2C, 2D), even at three and five days after TMZ treatment, when IDH1 mutant cells have already exited the G2 checkpoint (Figure 1D). These data indicate that IDH1 mutant astrocytes prematurely exit the G2 checkpoint with unrepaired DNA damage and that progression into mitosis is facilitated by checkpoint adaptation rather than checkpoint recovery.

\section{Inhibition of polo-like kinase 1 sensitizes IDH1 mutant astrocytes to TMZ}

PLK1 regulates G2 checkpoint adaptation and progression into mitosis following DNA damage and acts antagonistically to the CHK1 signaling pathway which is essential for maintenance of G2 checkpoint arrest [31-35]. Aberrant PLK1 activation can facilitate G2 checkpoint bypass and repress apoptotic signaling pathways, allowing cells to divide and survive despite failing to complete repair of damaged DNA [24, 36, 37]. One mechanism by which PLK1 inactivates the G2 DNA damage checkpoint is through phosphorylation of the CHK1 regulatory protein Claspin, which targets it for degradation and leads to the inactivation of the ATR/CHK1 signaling pathway [34]. Examination of PLK1 and CHK1 phosphorylation in TMZ-treated cells revealed elevated PLK1 activation and diminished CHK1 activation in the IDH1 mutant astrocytes (Figure 3A).

Several PLK1 inhibitors are currently in clinical trials as cancer therapeutics [25]. To determine if inhibition of PLK1 sensitizes IDH1 mutant astrocytes to TMZ, we treated cells with BI2536, a potent and selective PLK1 inhibitor. Co-treatment of IDH1 mutant cells with TMZ and BI2536 resulted in more than a threefold decrease in clonogenic survival rate when compared to TMZ or BI2536 treatment alone $(\mathrm{P}<0.001)$ (Figure $3 \mathrm{~B})$. Compared to TMZ alone, treatment with BI2536 and TMZ also increased CHK1 activation at day 3 post TMZ (Figure 3C). Combination treatment of IDH1 WT cells resulted in a less dramatic, though significant reduction in clonogenic survival when compared to treatments of either TMZ or BI2536 alone (Supplementary Figure 2).
A.
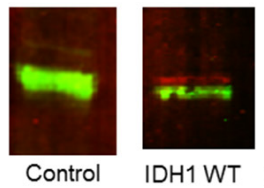

IDH1WT

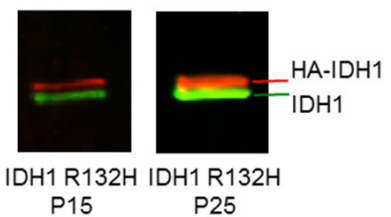

D. Days post TMZ Control

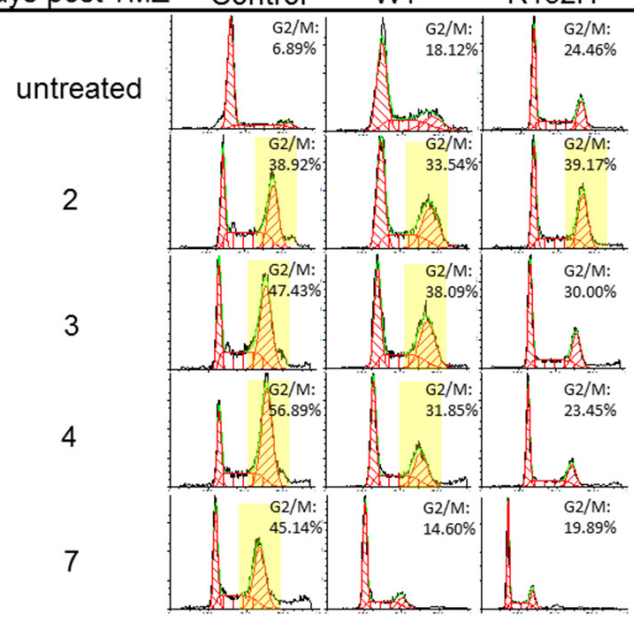

B.

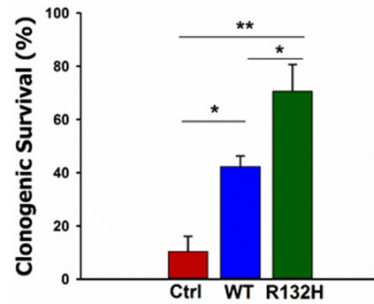

C.

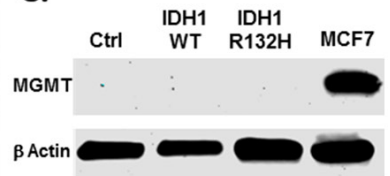

E.

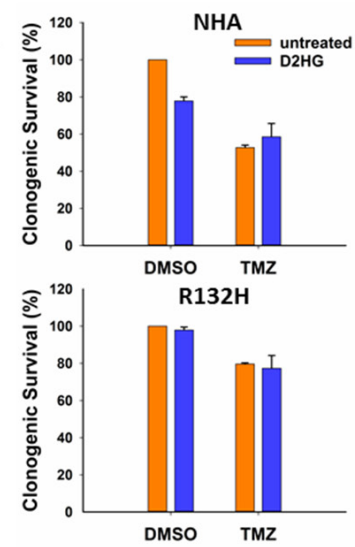

Figure 1: IDH1 mutation promotes resistance to TMZ by D2HG production. A. Western blot confirming expression of exogenous HA-IDH1 (red) and endogenous IDH1 (green). B. Clonogenic survival of empty vector control, IDH1 WT, and IDH1 mutant astrocytes after treatment with $100 \mu \mathrm{M}$ TMZ. C. MGMT expression was not detectable by Western blot in astrocytes regardless of IDH1 status. MCF7 cells were used as a positive control. D. Impact of mutant IDH1 on cell cycle profiles in response to TMZ treatment. Yellow boxes indicate $>30 \%$ of cells in G2/M. E. Clonogenic survival of parental astrocytes (top) and IDH1 mutant astrocytes (bottom) cultured with or without $5 \mathrm{mM}$ D2HG and treated with TMZ. There was a statistically significant interaction between D2HG and TMZ treatments in the NHA ( $\mathrm{P}=0.02)$ but not in IDH1 mutant astrocytes. Error bars represent SEM. $\mathrm{P}<0.05(*) ; \mathrm{P}<0.01(* *)$. 

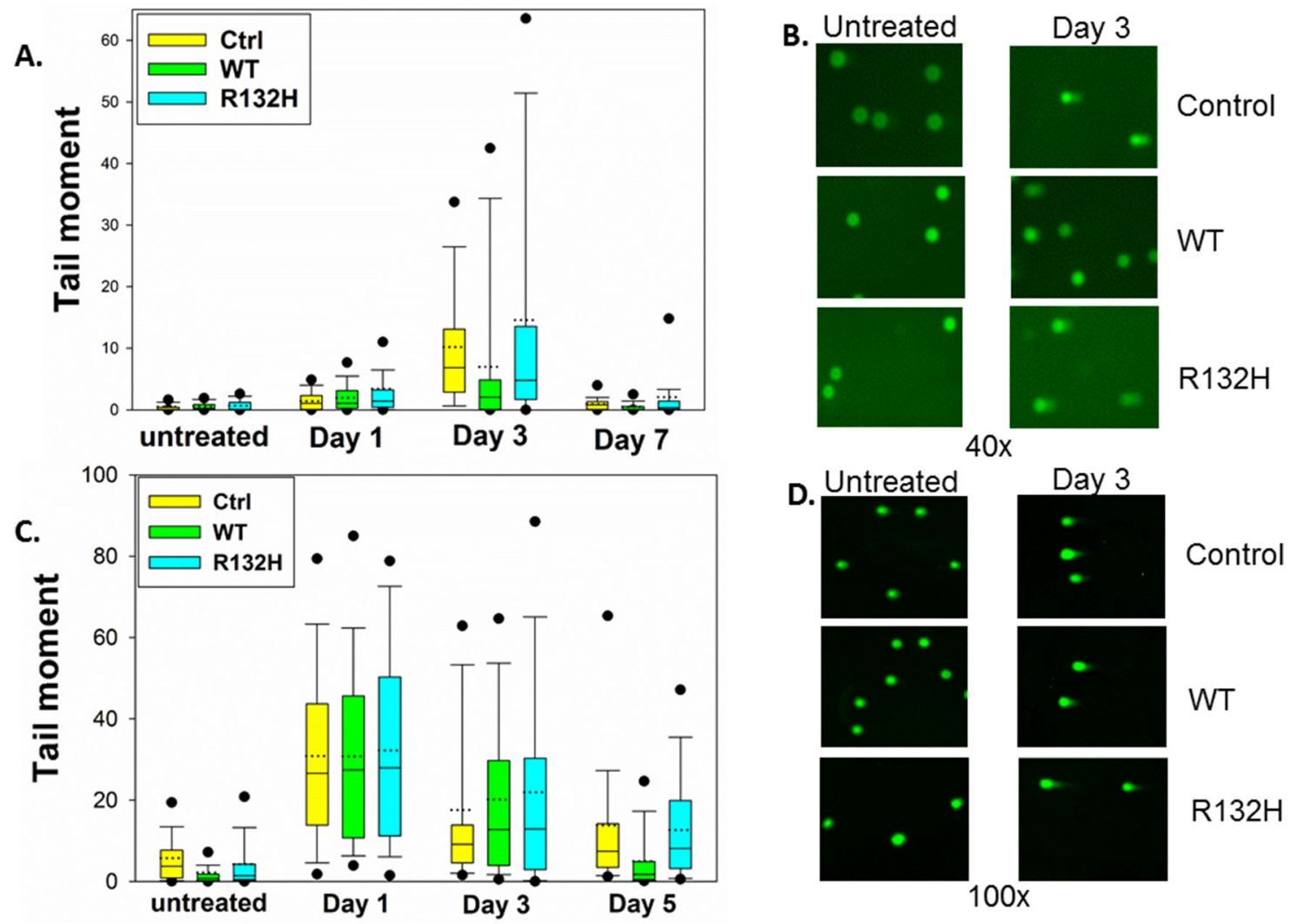

Figure 2: IDH1 mutation does not affect levels of DNA damage following TMZ treatment. A. Alkaline comet assay reveals no significant difference in total DNA damage between control, IDH1 WT, or IDH1 mutant astrocytes at day 1, 3, or 7 post TMZ treatment. B. Representative images of alkaline comet assay three days after TMZ treatment. C. No significant difference in double-strand DNA breaks was detected by neutral comet assay. D. Representative images of neutral comet assay three days post TMZ. Box plots represent median, first, and third quartiles. Bars show $10^{\text {th }}$ and $90^{\text {th }}$ percentiles. Circles show $5^{\text {th }}$ and $95^{\text {th }}$ percentiles.

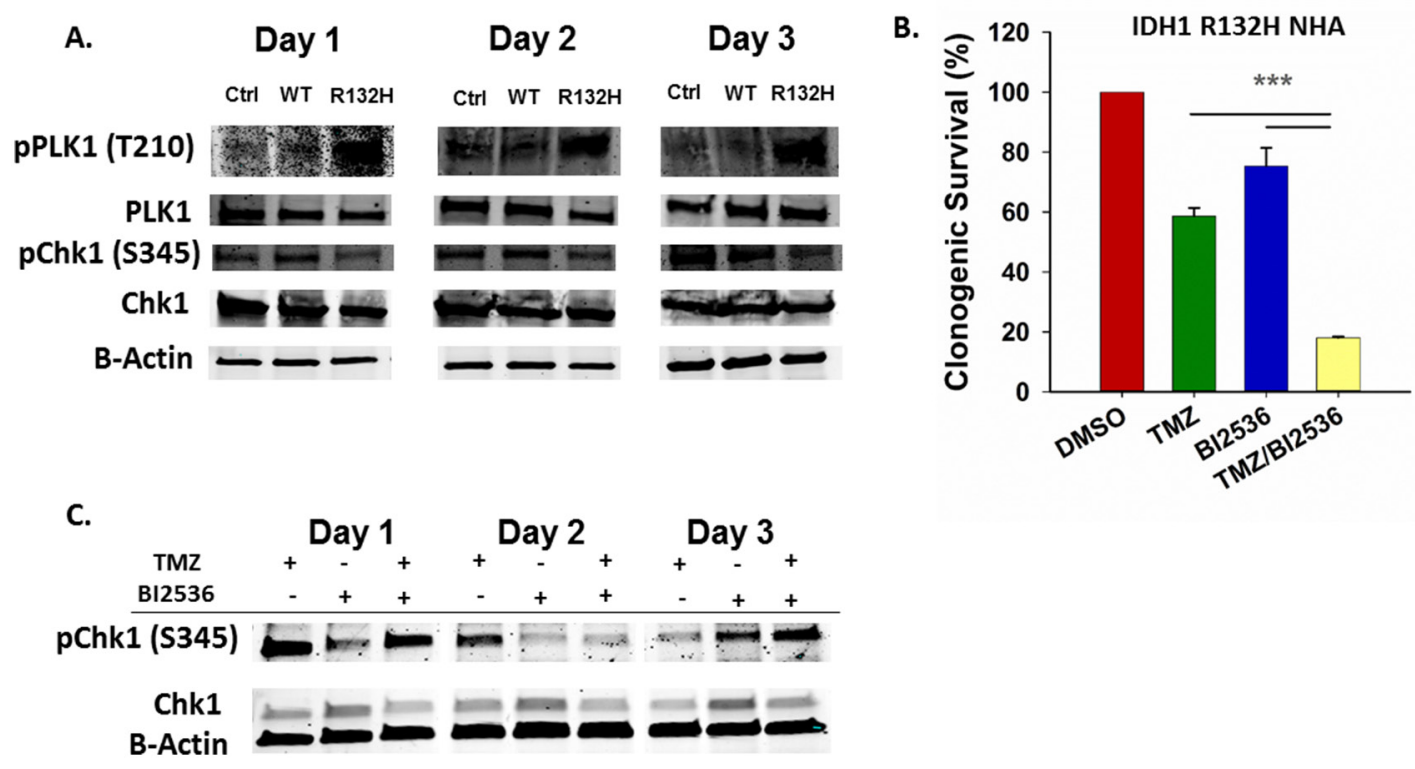

Figure 3: Inhibition of PLK1 sensitizes IDH1 mutant cells to TMZ. A. Western blot using phospho-specific antibodies shows increased PLK1 activation and decreased CHK1 activation in mutant IDH1 astrocytes after TMZ treatment compared to control and WT astrocytes. B. Clonogenic survival of IDH1 mutant astrocytes following treatment with TMZ and a PLK1 inhibitor, BI2536. C. Western blot for total and activate CHK1 in IDH1 mutant astrocytes treated with TMZ and BI2536. Error bars represent SEM. $\mathrm{n}=3$. P $<0.001(* * *)$. 


\section{A new in vivo model of IDH1 mutant glioma}

Since inhibition of PLK1 sensitized IDH1 mutant NHA to TMZ in vitro, we sought to determine whether a combination of TMZ and BI2536 is more effective than either single drug in vivo. However, cells from the majority of IDH1 mutant gliomas do not grow in vitro and tumors passaged directly in mice do not consistently retain their original characteristics [38-40]. We assessed the tumorigenic potential of the IDH1 mutant astrocytes in subcutaneous and orthotopic xenograft mouse models. Astrocytes expressing mutant $I D H 1$ for either 15, 25, or 50 passages (P15, P25, P50, respectively) were injected subcutaneously in mice. The astrocytes were all of approximately the same total passage number, and differed only in the number of passages with the IDHI mutant gene. All mice injected with the IDH1 mutant cells formed tumors. However, the time to tumor formation depended on the number of passages for which the cells expressed mutant IDH1 (Supplementary Figure 3A). The P50 cells formed tumors by 11 weeks, while P25 cells averaged almost 13 weeks, and P15 cells averaged more than 18 weeks to form tumors (Supplementary Figure 3A, 3B). Targeted sequencing revealed retention of wild-type and mutant $I D H 1$ alleles in the tumors (Supplementary Figure $3 C)$.

P50 cells were also transduced with a constitutively active luciferase reporter and tested for orthotopic tumorigenicity in the mouse brain. The first tumor was detectable by six weeks after injection and was fatal by week 9 (Supplementary Figure 3D). Of 12 mice injected, only three formed tumors that were clearly detectable by luminescence. We therefore chose to test the therapeutic treatments in the subcutaneous model.

\section{BI2536 enhances TMZ anti-tumor efficacy in vivo}

After establishing that IDH1 mutant astrocytes form tumors efficiently as subcutaneous xenografts, we tested TMZ and BI2536 as a combination treatment for subcutaneous, IDH1 mutant tumors in NOD-scid IL2Rgamma ${ }^{\text {null }}$ mice. Mice were injected twice, three days apart, with $80 \mathrm{mg} / \mathrm{kg}$ of TMZ and $40 \mathrm{mg} / \mathrm{kg} \mathrm{BI} 2536$ either alone or in combination, or with vehicle. Tumor volumes were tracked for 28 days. The combination therapy had significantly greater anti-tumor efficacy than TMZ or BI2536 as monotherapies (day $28 \mathrm{P}<0.001$ ). Treatment with the combination of both drugs produced a remarkable reduction in tumor size. At day 28, tumors in TMZ or BI2536 treated mice were similar in size to those of the vehicle treated mice (Figure 4A, 4B). Notably, five of eight mice treated with both drugs exhibited complete tumor regression. In the three cases where tumor regression was not complete, the tumor shrank markedly (Figure 4B), with no tumor exceeding $20 \mathrm{~mm}^{3}$ at day 28 .
In contrast, each of the other three treatment groups had an average tumor volume exceeding $900 \mathrm{~mm}^{3}$ at day 28 (Figure 4A). Mice receiving the combination treatment showed no obvious signs of toxicity and lost no more than $10 \%$ body weight at any point in time (Supplementary Figure 4).

\section{PLK1 inhibitor plus TMZ combination is effective in IDH1 mutant patient-derived cells}

After testing the combination therapy in IDH1 mutant astrocytes, we were able to obtain GBM164 cells which are patient derived cells that are maintained as xenografts but can be cultured for several passages in vitro [41]. The cells were genotyped and confirmed to be IDH1 heterozygous mutant (Supplementary Figure 5A). To confirm that the effect of combination treatment with BI2536 and TMZ is not unique to our astrocyte model, a cell viability assay was performed on GBM164 cells. Combination treatment resulted in over a seven fold decrease in cell viability compared to TMZ $(\mathrm{P} \leq 0.001)$ or BI2536 ( $\mathrm{P}=0.002)$ alone (Supplementary Figure 5B).

\section{DISCUSSION}

In gliomas, when the $I D H 1$ mutation is present, it is typically found throughout the entirety of otherwise heterogeneous tumors, which makes targeting vulnerabilities conferred by mutant $I D H I$ very appealing [5]. In the current work, we report that the IDHI mutation promotes TMZ resistance through G2 checkpoint adaptation facilitated by PLK1 activation. Additionally, treatment with a PLK1 inhibitor dramatically improves TMZ efficacy while establishing the use of IDHI mutant astrocytes in a xenograft mouse model.

Our data indicate that in the context of an $I D H I$ mutation, PLK1 activation promotes bypass of the TMZ-induced DNA damage checkpoint, limiting TMZ effectiveness. PLK1 can inactivate the DNA damage checkpoint by inactivating or facilitating degradation of target proteins such as Claspin, a mediator of CHK1 activation and G2 checkpoint maintenance [34, 35]. CHK1 and PLK1 act antagonistically as PLK1 can be inactivated in a CHK1 activity-dependent manner [31, 32]. Consistent with this model, our data confirm that CHK1 is inactivated and PLK1 is activated in IDH1 mutant cells following TMZ exposure.

Interestingly, TMZ resistance has been linked to IDH1 mutant-mediated transformation, rather than the immediate activity of the mutant enzyme [23]. Clearly, the long-term and immediate effects of IDH1 mutation are not mutually exclusive and while the indirect effects of an IDH1 mutation likely play a role, we show that D2HG also acutely promotes $\mathrm{TMZ}$ resistance.

While the mechanism of PLK1 activation in IDHI mutant tumors is still under investigation, we postulate 
that the immediate effect may be in part through the inhibition of $\alpha \mathrm{KG}$-dependent enzymes. D2HG produced by mutant IDH1 inhibits members of the TET and JmjC families of enzymes, which are regulators of DNA and histone methylation, respectively. TET inhibition is associated with DNA hypermethylation resulting in the $\mathrm{CpG}$ island methylation phenotype and altered gene expression profile. However, no significant changes in expression or DNA methylation occur at $P L K 1$ or $C H K 1$ loci in the $I D H 1$ mutant astrocytes [27]. Additionally, genes for upstream regulators of PLK1 and CHK1 such as Aurora A, Bora, and ATR also remain unaffected. A single $\mathrm{CpG}$ island locus in the ATRIP gene was reported to be slightly hypomethylated, though gene expression is not significantly affected [27].

Alternatively, it is possible that $\mathrm{D} 2 \mathrm{HG}$ promotes PLK1 activation by inhibiting Egln3 activity. Hydroxylation of Telo 2 by the $\alpha \mathrm{KG}$-dependent dioxygenase Egln3 is required for activation of the ATR/ CHK1 checkpoint pathway which in turn leads to PLK1 inactivation [42, 43]. However, D2HG competes with $\alpha \mathrm{KG}$ as an Egln3 substrate, possibly leading to inhibition of Egln 3 activity and ultimately to CHK1 inactivation and PLK1 activation. Inhibition of Egln 3 and the corresponding decrease in Telo 2 hydroxylation has also been shown to decrease apoptosis following DNA damage [42].

$\alpha \mathrm{KG}$-dependent dioxygenases may also account for the mild temozolomide (TMZ) resistance in the IDH1 WT NHA. While IDH1 mutant NHA display hypermethylation as a result of TET inhibition, Turcan et al. reported genomic hypomethylation in IDH1 WT NHA suggesting that $\alpha \mathrm{KG}$ production may enhance $\alpha \mathrm{KG}$-dependent dioxygenase activity [27]. It is possible that IDH1 WT NHA repair TMZ-induced DNA damage more efficiently by activating EGLN3 or members of the AlkBH family

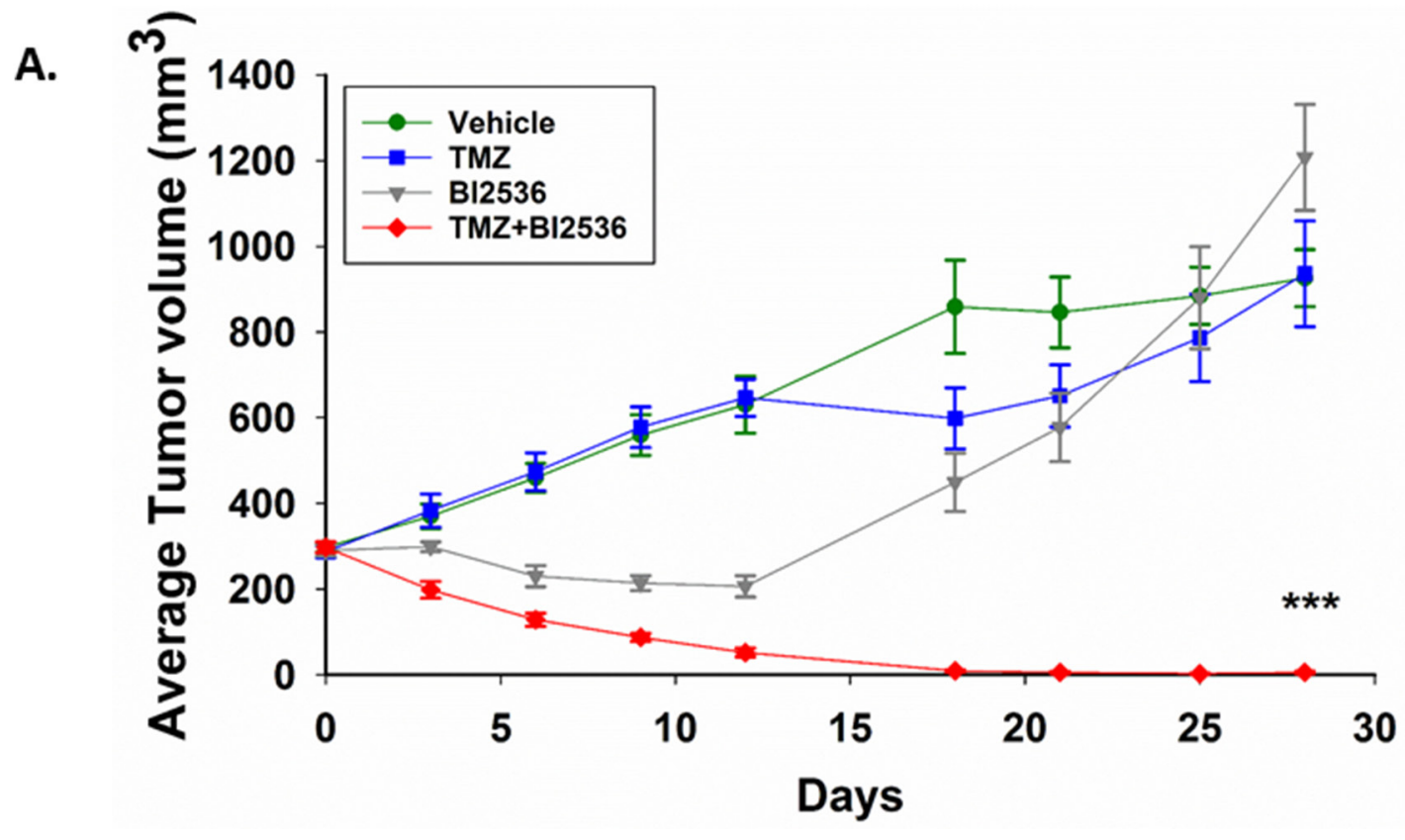

B.

untreated

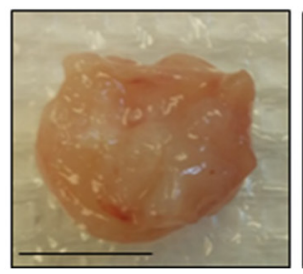

TMZ

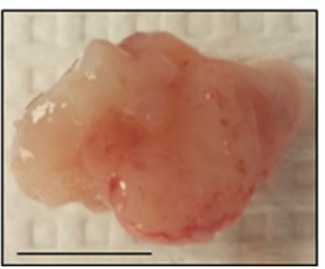

BI2536

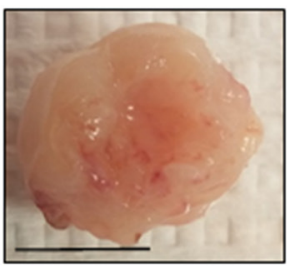

$\mathrm{TMZ}+\mathrm{BI} 2536$

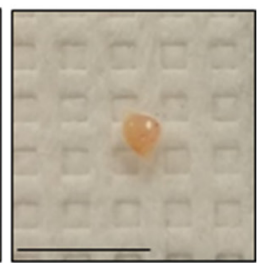

Figure 4: Combination of TMZ and BI2536 leads to a marked tumor regression in IDH1 mutant tumors. A. Average subcutaneous tumor volumes of vehicle, TMZ, BI2536, and TMZ+BI2536-treated mice over 28 days. B. Representative images of excised subcutaneous tumors 31 days after treatment began. Scale bars $=1 \mathrm{~cm}$. Error bars represent $\mathrm{SEM}$. $\mathrm{P}<0.001(* * *)$. $\mathrm{n} \geq 5$ mice per treatment group. 
of $\alpha \mathrm{KG}$-dependent DNA repair enzymes. Improved DNA repair capacity in the IDH1 WT NHA would account for the shortened G2 arrest (Figure 1D) and fewer double strand breaks at day 5 post TMZ (Figure 2C).

Increased homologous recombination has also been proposed as the mechanism of TMZ resistance in mutant IDH1-transformed astrocytes [23]. However, we demonstrate that $I D H 1$ mutant cells have no less DNA damage than IDH1 WT and control cells, indicating checkpoint adaptation rather than recovery is responsible for early G2 checkpoint exit. While PLK1 has been shown to phosphorylate BRCA1 and RAD51 to promote homologous recombination, $[44,45]$ it is possible that the IDH1 mutation and PLK1 activation not only promote homologous recombination, or at least activation of the homologous recombination machinery, but also premature mitotic progression prior to complete repair of damaged DNA.

To date, all work concerning the use of temozolomide (TMZ) in combination with a PLK1 inhibitor has been in the context of primary grade IV glioblastoma, which rarely carries an IDH1 mutation [46-48]. Compared to their wild type counterparts, IDH1 mutant gliomas have a favorable clinical response to TMZ and IDH1 has become an important prognostic factor [49]. Importantly, IDH1 wild-type and mutant gliomas are molecularly very different and comparative clinical outcome does not indicate how mutant IDH1 affects a tumor's response to treatment. Additionally, our work demonstrates the context-dependent effects of mutant $I D H 1$ which has previously been reported to either increase or have no effect on TMZ sensitivity when expressed in U87 and U373 cell lines [50, 51]. In contrast, we and others have shown that the expression of mutant IDH1 in untransformed cells promotes cellular transformation and temozolomide (TMZ) resistance [23].

The sensitivity of IDHI mutant tumors to a PLK1 inhibitor plus TMZ combination may have implications beyond glioma treatment. IDH mutation is found frequently in acute myeloid leukemia, cholangiocarcinoma, osteosarcoma, and in central and periosteal chondrosarcomas which are nonresponsive to current chemotherapy regimens [52]. Interestingly, a variety of PLK1 inhibitors are in clinical and preclinical development with two of them in late clinical trials [25]. Our findings establish PLK1 as a very promising target in IDH1 mutant tumors and warrant consideration of clinical trials for TMZ and PLK1 inhibitor combinations.

\section{MATERIALS AND METHODS}

\section{Cell lines}

Immortalized human astrocytes have been described elsewhere [26]. HA-tagged wild-type and R132H mutant IDH1 were cloned into pBABE-neo retroviral vector.
Astrocytes were transduced with retrovirus and underwent G418 selection. Clones were screened for expression of HA-IDH1. IDH1 mutant astrocytes used at passage 50 were generated as previously described [27].

GBM164, MCF7, and all NHA cell lines were grown as adherent cells in DMEM, 10\% FBS.

\section{Western blotting}

Western blotting was performed using Bio-Rad mini-protean ${ }^{\circledR} \mathrm{TGX}^{\mathrm{TM}}$ gels and PVDF membranes. Primary antibodies used: anti-IDH1 (N-20;\#sc-49996, Santa Cruz), anti-pPLK1 T210 (D5H7;\#9062, Cell Signaling), anti-PLK1 (208G4, \#4513, Cell Signaling), anti-pCHK1 S345 (133D3;\#2348, Cell Signaling), antiCHK1 (2G1D5, \#2360, Cell Signaling), anti- $\beta$ Actin (8H10D10, \#3700, Cell Signaling), anti-HA (6E2, \#2367, Cell Signaling), and anti-MGMT (OAAF03046, Aviva Systems Biology). Imaging was performed on a LI-COR Odyssey imager.

\section{Nuclear magnetic resonance spectroscopy}

Cell collections and extractions were performed as previously described [53]. Briefly, the hydrophilic cell extract samples were dried in a SpeedVac centrifuge then resuspended in NMR buffer. One-dimensional ${ }^{1} \mathrm{H}$ NMR spectra were recorded using Carr-Purcell-Meiboon-Gill pulse sequence with presaturation of the water peak on a $600 \mathrm{MHz}$ INOVA spectrometer. Experiments were run with 4 dummy scans and 128 acquisition scans (acquisition time: $2.09 \mathrm{~s}$, relaxation delay: $4.0 \mathrm{~s}$, mixing time: $60 \mathrm{~ms}$ ). Spectral width was $26 \mathrm{ppm}$, and $64 \mathrm{~K}$ real data points were collected. Pure D2HG was used to confirm the metabolite of interest. All NMR data were processed using TopSpin3.1 (Bruker Analytik, Rheinstetten, Germany). All FIDs were subjected to exponential line-broadening of $0.3 \mathrm{~Hz}$. Upon Fourier transformation, each spectrum was manually phased, baseline corrected, and referenced to the internal standard TMSP at $0.0 \mathrm{ppm}$.

\section{Clonogenic survival assay}

Cells $\left(1 \times 10^{3}\right)$ were plated on $10 \mathrm{~cm}$ plates. When adhered, cells were treated with DMSO or 2nM BI2536 (Selleck) for $42 \mathrm{hrs}, 100 \mu \mathrm{M}$ temozolomide (Cayman) for $18 \mathrm{hrs}$, or $100 \mu \mathrm{M} \mathrm{TMZ}+2 \mathrm{nM}$ BI 2536 for $18 \mathrm{hrs}$ followed by an additional 24hrs with 2nM BI2536 so that BI2536 was present during the second cell cycle following TMZ exposure. The day after TMZ was washed out was designated day 1 post TMZ. When D2HG treatment was included, cells were incubated with complete media plus $5 \mathrm{mM}$ D2HG from 8hrs prior to TMZ treatment until cells were fixed and stained. Cells were then grown in DMEM, $10 \%$ FBS until colonies clearly formed in DMSOtreated plates. The plates were washed, fixed with $4 \%$ paraformaldehyde, and stained with $0.1 \%$ crystal violet. 


\section{Cell viability assay}

In a 96 well plate, $5 \times 10^{3}$ GBM164 cells were plated per well. Two days later cells were treated with $200 \mu \mathrm{M}$ TMZ and 4nM BI2536 and incubated for three days, after which treatment was washed out and cell viability was measured using cell counting kit 8 (Dojindo Laboratories, Rockville, MD) according to the manufacturer's protocol.

\section{Propidium iodide staining/cell cycle analysis}

After 18hrs TMZ treatment, cells were washed and incubated with complete media until collection. Cells were trypsinized, washed, fixed with cold $70 \%$ ethanol, and propidium iodide stained. Flow cytometry was performed on a BD-LSR-Fortessa flow cytometer. Analysis was performed on FCS Express 4 with $\geq 10^{4}$ events per time point.

\section{Comet assay}

The Trevigen Comet assay kit (4250-050-K) was used according to manufacturer's protocol. All samples for a given time point were run together in duplicate. DNA was stained with SYBR Gold (LifeTechnologies), viewed with an Olympus-BX51 microscope, imaged with a SPOTRT-KE camera (Diagnostic Instruments), and $\geq 40$ comets were scored for each sample using OpenComet [54].

\section{Testing drug efficacy in vivo}

Five-week-old female NOD-scid IL2Rgamma ${ }^{\text {null }}$ mice were injected with $2.5 \times 10^{6}$ cells subcutaneously in the right flanks. Treatments were administered when tumors reached $300 \mathrm{~mm}^{3}$, and again three days later. Size was determined with calipers and volume was calculated by: $\mathrm{V}=\mathrm{LxWxH} / 2$. Temozolomide $(80 \mathrm{mg} / \mathrm{kg})$ was injected intraperitoneally in $10 \%$ DMSO. BI2536 $(40 \mathrm{mg} / \mathrm{kg})$ was dissolved in $0.02 \mathrm{~N} \mathrm{HCl}$ and administered by tail vein injection.

\section{Intracranial xenograft}

Intracranial xenografts were established as previously described [55]. Briefly, five-week-old female NOD-scid IL2Rgamma ${ }^{\text {null }}$ mice were anesthetized, fixed in a stereotactic apparatus, and $10^{5}$ cells constitutively expressing luciferase were injected with a Hamilton syringe $2 \mathrm{~mm}$ lateral to the bregma point at a depth of $3 \mathrm{~mm}$. Mice were imaged with a Bruker In-Vivo MS FX PRO. All mouse work was in accordance with a protocol approved by the University of Cincinnati Institutional Animal Care and Use Committee.

\section{Statistical analysis}

Significance was set to $\mathrm{P} \leq 0.05$ for all experiments. One-way ANOVA was used to compare between groups.
To test for treatment interactions, two way ANOVA was used. The Holm-Sidak method was used for post-hoc testing. All statistical analyses were performed with SigmaPlotV13.

\section{ACKNOWLEDGMENTS}

We would like to thank Dr. Jann Sarkaria from Mayo Clinic for generously providing the GBM164 cell line, Dr. Changchun Xie from the Division of Biostatistics and Bioinformatics, Department of Environmental Health at the University of Cincinnati for his help with statistical analysis, Kathleen LaSance from the University of Cincinnati imaging core for her assistance with in vivo imaging, and Dr. Peter Stambrook from the Department of Molecular Genetics at the University of Cincinnati for the critical reading of this manuscript.

\section{CONFLICTS OF INTEREST}

With regard to the data presented in this manuscript, the authors declare no conflicts of interest. The funding agencies had no involvement in the design of the experiments or in data interpretation.

\section{FUNDING SUPPORT}

Funding for this project was provided by the University of Cincinnati, the UC Brain Tumor Center, and the National Center for Advancing Translational Sciences of the National Institutes of Health, award number 1UL1TR001425-01 (EMB). This work was also supported in part by 1R01CA158372-01A0 (XQ).

\section{REFERENCES}

1. Smoll NR, Hamilton B. Incidence and relative survival of anaplastic astrocytomas. Neuro Oncol. 2014; 16: 1400-7. doi: 10.1093/neuonc/nou053.

2. Omuro A, DeAngelis LM. Glioblastoma and other malignant gliomas: a clinical review. JAMA. 2013; 310:1842-50. doi: 10.1001/jama.2013.280319.

3. Louis DN, Ohgaki H, Wiestler OD, Cavenee WK, Burger PC, Jouvet A, Scheithauer BW, Kleihues P. The 2007 WHO classification of tumours of the central nervous system. Acta Neuropathol. 2007; 114:97-109. doi: 10.1007/ s00401-007-0243-4.

4. Yan H, Parsons DW, Jin G, McLendon R, Rasheed BA, Yuan W, Kos I, Batinic-Haberle I, Jones S, Riggins GJ, Friedman H, Friedman A, Reardon D, et al. IDH1 and IDH2 mutations in gliomas. N Engl J Med. 2009; 360:765-73. doi: 10.1056/NEJMoa0808710.

5. Lass U, Nümann A, von Eckardstein K, Kiwit J, Stockhammer F, Horaczek JA, Veelken J, Herold-Mende C, Jeuken J, von Deimling A, Mueller W. Clonal analysis in recurrent astrocytic, oligoastrocytic and oligodendroglial 
tumors implicates IDH1- mutation as common tumor initiating event. Lesniak MS, editor. PLoS One. Public Library of Science; 2012; 7: e41298. doi: 10.1371/journal. pone.0041298.

6. Dang L, White DW, Gross S, Bennett BD, Bittinger MA, Driggers EM, Fantin VR, Jang HG, Jin S, Keenan MC, Marks KM, Prins RM, Ward PS, et al. Cancer-associated IDH1 mutations produce 2-hydroxyglutarate. Nature. 2009; 462: 739-44. doi: 10.1038/nature08617.

7. Xu W, Yang H, Liu Y, Yang Y, Wang P, Kim S-H, Ito S, Yang C, Wang P, Xiao M-T, Liu L, Jiang W, Liu J, et al. Oncometabolite 2-hydroxyglutarate is a competitive inhibitor of $\alpha$-ketoglutarate-dependent dioxygenases. Cancer Cell. 2011; 19:17-30. doi: 10.1016/j. ccr.2010.12.014.

8. Chowdhury R, Yeoh KK, Tian Y-M, Hillringhaus L, Bagg EA, Rose NR, Leung IKH, Li XS, Woon ECY, Yang M, McDonough MA, King ON, Clifton IJ, et al. The oncometabolite 2-hydroxyglutarate inhibits histone lysine demethylases. EMBO Rep. EMBO Press; 2011; 12:463-9. doi: 10.1038/embor.2011.43.

9. Stupp R, Hegi ME, Gilbert MR, Chakravarti A. Chemoradiotherapy in malignant glioma: standard of care and future directions. J Clin Oncol. 2007; 25:4127-36. doi: 10.1200/JCO.2007.11.8554.

10. Zhang J, Stevens MFG, Bradshaw TD. Temozolomide: mechanisms of action, repair and resistance. Curr Mol Pharmacol. 2012; 5:102-14.

11. Hirose Y, Berger MS, Pieper RO. p53 Effects Both the Duration of G2/M Arrest and the Fate of Temozolomidetreated Human Glioblastoma Cells. Cancer Res. 2001; 61:1957-63.

12. Mulholland S, Pearson DM, Hamoudi RA, Malley DS, Smith CM, Weaver JMJ, Jones DTW, Kocialkowski S, Bäcklund LM, Collins VP, Ichimura K. MGMT CpG island is invariably methylated in adult astrocytic and oligodendroglial tumors with IDH1 or IDH2 mutations. Int J Cancer. 2012; 131:1104-13. doi: 10.1002/ijc.26499.

13. Houillier C, Wang X, Kaloshi G, Mokhtari K, Guillevin R, Laffaire J, Paris S, Boisselier B, Idbaih A, LaigleDonadey F, Hoang-Xuan K, Sanson M, Delattre J-Y. IDH1 or IDH2 mutations predict longer survival and response to temozolomide in low-grade gliomas. Neurology. 2010; 75: 1560-6. doi: 10.1212/WNL.0b013e3181f96282.

14. SongTao Q, Lei Y, Si G, YanQing D, HuiXia H, XueLin Z, LanXiao W, Fei Y. IDH mutations predict longer survival and response to temozolomide in secondary glioblastoma. Cancer Sci. 2012; 103:269-73. doi: 10.1111/j.1349-7006.2011.02134.x.

15. Gil Del Alcazar CR, Todorova PK, Habib AA, Mukherjee B, Burma S. Augmented HR Repair Mediates Acquired Temozolomide Resistance in Glioblastoma. Mol Cancer Res. 2016; 14:928-40. doi: 10.1158/1541-7786. MCR-16-0125.
16. McFaline-Figueroa JL, Braun CJ, Stanciu M, Nagel ZD, Mazzucato P, Sangaraju D, Cerniauskas E, Barford K, Vargas A, Chen Y, Tretyakova N, Lees JA, Hemann MT, et al. Minor Changes in Expression of the Mismatch Repair Protein MSH2 Exert a Major Impact on Glioblastoma Response to Temozolomide. Cancer Res. 2015; 75:312738. doi: 10.1158/0008-5472.CAN-14-3616.

17. Palma JP, Wang YC, Rodriguez LE, Montgomery D, Ellis PA, Bukofzer G, Niquette A, Liu X, Shi Y, Lasko L, Zhu GD, Penning TD, Giranda VL, et al. ABT- 888 confers broad in vivo activity in combination with temozolomide in diverse tumors. Clin Cancer Res. 2009; 15:7277-90. doi: 10.1158/1078-0432.CCR-09-1245.

18. Roos WP, Nikolova T, Quiros S, Naumann SC, Kiedron O, Zdzienicka MZ, Kaina B. Brca2/Xrcc2 dependent HR, but not NHEJ, is required for protection against $\mathrm{O}(6)$ methylguanine triggered apoptosis, DSBs and chromosomal aberrations by a process leading to SCEs. DNA Repair (Amst). 2009; 8:72-86. doi: 10.1016/j.dnarep.2008.09.003.

19. Brat DJ, Verhaak RGW, Aldape KD, Yung WKA, Salama SR, Cooper LAD, Rheinbay E, Miller CR, Vitucci M, Morozova O, Robertson AG, Noushmehr H, Laird PW, et al. Comprehensive, Integrative Genomic Analysis of Diffuse Lower-Grade Gliomas. N Engl J Med. 2015; 372:2481-98. doi: 10.1056/NEJMoa1402121.

20. Minniti G, Scaringi C, Arcella A, Lanzetta G, Di Stefano D, Scarpino S, Bozzao A, Pace A, Villani V, Salvati M, Esposito V, Giangaspero F, Enrici RM. IDH1 mutation and MGMT methylation status predict survival in patients with anaplastic astrocytoma treated with temozolomide-based chemoradiotherapy. J Neurooncol. 2014; 118:377-83. doi: 10.1007/s11060-014-1443-0.

21. Villani V, Merola R, Vidiri A, Fabi A, Carosi M, Giannarelli D, Marucci L, Maschio M, Carapella CM, Pace A. Temozolomide low-dose chemotherapy in newly diagnosed low-grade gliomas: activity, safety, and longterm follow-up. Tumori. 2016. doi: 10.5301/tj.5000565.

22. Johnson BE, Mazor T, Hong C, Barnes M, Aihara K, McLean CY, Fouse SD, Yamamoto S, Ueda H, Tatsuno K, Asthana S, Jalbert LE, Nelson SJ, et al. Mutational analysis reveals the origin and therapy-driven evolution of recurrent glioma. Science. 2014; 343:189-93. doi: 10.1126/ science. 1239947.

23. Ohba S, Mukherjee J, See WL, Pieper RO. Mutant IDH1driven cellular transformation increases RAD51-mediated homologous recombination and temozolomide resistance. Cancer Res. 2014; 0008-5472.CAN-14-0924-. doi: 10.1158/0008-5472.CAN-14-0924.

24. Bahassi EM. Polo-like kinases and DNA damage checkpoint: beyond the traditional mitotic functions. Exp Biol Med (Maywood). SAGE Publications; 2011; 236:64857. doi: 10.1258/ebm.2011.011011.

25. Talati C, Griffiths EA, Wetzler M, Wang ES. Polo-like kinase inhibitors in hematologic malignancies. Crit 
Rev Oncol Hematol. 2016; 98:200-10. doi: 10.1016/j. critrevonc.2015.10.013.

26. Sonoda Y, Ozawa T, Hirose Y, Aldape KD, McMahon M, Berger MS, Pieper RO. Formation of Intracranial Tumors by Genetically Modified Human Astrocytes Defines Four Pathways Critical in the Development of Human Anaplastic Astrocytoma. Cancer Res. 2001; 61:4956-60.

27. Turcan S, Rohle D, Goenka A, Walsh LA, Fang F, Yilmaz E, Campos C, Fabius AWM, Lu C, Ward PS, Thompson CB, Kaufman A, Guryanova O, et al. IDH1 mutation is sufficient to establish the glioma hypermethylator phenotype. Nature. Nature Publishing Group; 2012; 483:479-83. doi: 10.1038/ nature 10866.

28. Ma S, Jiang B, Deng W, Gu Z-K, Wu F-Z, Li T, Xia Y, Yang H, Ye D, Xiong Y, Guan K-L. D-2-hydroxyglutarate is essential for maintaining oncogenic property of mutant IDH-containing cancer cells but dispensable for cell growth. Oncotarget. 2015; 6:8606-20. doi: 10.18632/ oncotarget.3330.

29. Gilbert MR, Liu Y, Neltner J, Pu H, Morris A, Sunkara M, Pittman T, Kyprianou N, Horbinski C. Autophagy and oxidative stress in gliomas with IDH1 mutations. Acta Neuropathol. 2014; 127:221-33. doi: 10.1007/ s00401-013-1194-6.

30. Andronesi OC, Rapalino O, Gerstner E, Chi A, Batchelor TT, Cahill DP, Sorensen AG, Rosen BR. Detection of oncogenic IDH1 mutations using magnetic resonance spectroscopy of 2-hydroxyglutarate. Journal of Clinical Investigation. American Society for Clinical Investigation; 2013. p. 3659-63. doi: 10.1172/JCI67229.

31. Tsvetkov L, Stern DF. Phosphorylation of Plk1 at S137 and T210 is inhibited in response to DNA damage. Cell Cycle. 2005; 4:166-71.

32. Lee H-J, Hwang H-I, Jang Y-J. Mitotic DNA damage response: Polo-like kinase-1 is dephosphorylated through ATM-Chk1 pathway. Cell Cycle. 2010; 9:2389-98.

33. van Vugt MATM, Brás A, Medema RH. Polo-like Kinase-1 Controls Recovery from a G2 DNA Damage-Induced Arrest in Mammalian Cells. Mol Cell. 2004; 15:799-811. doi: 10.1016/j.molcel.2004.07.015.

34. Mamely I, van Vugt MATM, Smits VAJ, Semple JI, Lemmens B, Perrakis A, Medema RH, Freire R. Pololike kinase-1 controls proteasome-dependent degradation of Claspin during checkpoint recovery. Curr Biol. 2006; 16:1950-5. doi: 10.1016/j.cub.2006.08.026.

35. Liu Q, Guntuku S, Cui XS, Matsuoka S, Cortez D, Tamai K, Luo G, Carattini-Rivera S, DeMayo F, Bradley A, Donehower LA, Elledge SJ. Chk1 is an essential kinase that is regulated by Atr and required for the G(2)/M DNA damage checkpoint. Genes Dev. 2000; 14:1448-59.

36. Chun G, Bae D, Nickens K, O'Brien TJ, Patierno SR, Ceryak S. Polo-like kinase 1 enhances survival and mutagenesis after genotoxic stress in normal cells through cell cycle checkpoint bypass. Carcinogenesis. 2010; 31:785-93. doi: 10.1093/carcin/bgq014.

37. Koida N, Ozaki T, Yamamoto H, Ono S, Koda T, Ando K, Okoshi R, Kamijo T, Omura K, Nakagawara A. Inhibitory role of Plk1 in the regulation of p73-dependent apoptosis through physical interaction and phosphorylation. J Biol Chem. 2008; 283:8555-63. doi: 10.1074/jbc.M710608200.

38. Piaskowski S, Bienkowski M, Stoczynska-Fidelus E, Stawski R, Sieruta M, Szybka M, Papierz W, Wolanczyk M, Jaskolski DJ, Liberski PP, Rieske P. Glioma cells showing IDH1 mutation cannot be propagated in standard cell culture conditions. Br J Cancer. 2011; 104:968-70. doi: 10.1038/bjc.2011.27.

39. Luchman HA, Chesnelong C, Cairncross JG, Weiss S. Spontaneous loss of heterozygosity leading to homozygous $\mathrm{R} 132 \mathrm{H}$ in a patient-derived IDH1 mutant cell line. Neuro Oncol. 2013; 15:979-80. doi: 10.1093/neuonc/not064.

40. Borodovsky A, Salmasi V, Turcan S, Fabius AWM, Baia GS, Eberhart CG, Weingart JD, Gallia GL, Baylin SB, Chan TA, Riggins GJ. 5-azacytidine reduces methylation, promotes differentiation and induces tumor regression in a patient-derived IDH1 mutant glioma xenograft. Oncotarget. 2013; 4:1737-47. doi: 10.18632/oncotarget.1408.

41. Carlson BL, Pokorny JL, Schroeder MA, Sarkaria JN. Establishment, maintenance and in vitro and in vivo applications of primary human glioblastoma multiforme (GBM) xenograft models for translational biology studies and drug discovery. Curr Protoc Pharmacol. NIH Public Access; 2011; Chapter 14: Unit 14.16. doi: 10.1002/0471141755.ph1416s52.

42. Xie L, Pi X, Mishra A, Fong G, Peng J, Patterson C. PHD3dependent hydroxylation of HCLK2 promotes the DNA damage response. J Clin Invest. 2012; 122:2827-36. doi: 10.1172/JCI62374.

43. Rendtlew Danielsen JM, Larsen DH, Schou KB, Freire R, Falck J, Bartek J, Lukas J. HCLK2 is required for activity of the DNA damage response kinase ATR. J Biol Chem. 2009; 284:4140-7. doi: 10.1074/jbc.M808174200.

44. Yata K, Lloyd J, Maslen S, Bleuyard J-Y, Skehel M, Smerdon SJ, Esashi F. Plk1 and CK2 act in concert to regulate Rad51 during DNA double strand break repair. Mol Cell. 2012; 45:371-83. doi: 10.1016/j.molcel.2011.12.028.

45. Chabalier-Taste C, Brichese L, Racca C, Canitrot Y, Calsou P, Larminat F, Chabalier-Taste C, Brichese L, Racca C, Canitrot Y, Calsou P, Larminat F. Polo-like kinase 1 mediates BRCA1 phosphorylation and recruitment at DNA double-strand breaks. Oncotarget. 2016; 7:2269-83. doi: 10.18632/oncotarget.6825.

46. Pezuk JA, Brassesco MS, Morales AG, de Oliveira JC, de Paula Queiroz RG, Machado HR, Carlotti CG, Neder L, Scrideli CA, Tone LG. Polo-like kinase 1 inhibition causes decreased proliferation by cell cycle arrest, leading to cell death in glioblastoma. Cancer Gene Ther. Nature America, Inc.; 2013; 20:499-506. doi: 10.1038/cgt.2013.46. 
47. Shen Y, Li J, Nitta M, Futalan D, Steed T, Treiber JM, Taich Z, Stevens D, Wykosky J, Chen H-Z, Carter BS, Becher OJ, Kennedy R, et al. Orthogonal targeting of EGFRvIII expressing glioblastomas through simultaneous EGFR and PLK1 inhibition. Oncotarget. 2015; 6:11751-67. doi: 10.18632/oncotarget.3996.

48. Triscott J, Lee C, Hu K, Fotovati A, Berns R, Pambid M, Luk M, Kast RE, Kong E, Toyota E, Yip S, Toyota B, Dunn SE. Disulfiram, a drug widely used to control alcoholism, suppresses the self-renewal of glioblastoma and over-rides resistance to temozolomide. Oncotarget. 2012; 3:1112-23. doi: 10.18632/oncotarget.604.

49. Cairncross JG, Wang M, Jenkins RB, Shaw EG, Giannini C, Brachman DG, Buckner JC, Fink KL, Souhami L, Laperriere NJ, Huse JT, Mehta MP, Curran WJ. Benefit from procarbazine, lomustine, and vincristine in oligodendroglial tumors is associated with mutation of IDH. J Clin Oncol. 2014; 32:783-90. doi: 10.1200/JCO.2013.49.3726.

50. Li S, Chou AP, Chen W, Chen R, Deng Y, Phillips HS, Selfridge J, Zurayk M, Lou JJ, Everson RG, Wu K-C, Faull KF, Cloughesy T, et al. Overexpression of isocitrate dehydrogenase mutant proteins renders glioma cells more sensitive to radiation. Neuro Oncol. Oxford University Press; 2013; 15:57-68. doi: 10.1093/neuonc/nos261.

51. Wang $\mathrm{P}, \mathrm{Wu} \mathrm{J}, \mathrm{Ma} \mathrm{S}$, Zhang L, Yao J, Hoadley KA, Wilkerson MD, Perou CM, Guan K-L, Ye D, Xiong Y.
Oncometabolite D-2-Hydroxyglutarate Inhibits ALKBH DNA Repair Enzymes and Sensitizes IDH Mutant Cells to Alkylating Agents. Cell Rep. 2015; 13:2353-61. doi: 10.1016/j.celrep.2015.11.029.

52. Molenaar RJ, Radivoyevitch T, Maciejewski JP, van Noorden CJF, Bleeker FE. The driver and passenger effects of isocitrate dehydrogenase 1 and 2 mutations in oncogenesis and survival prolongation. Biochim Biophys Acta - Rev Cancer. 2014; 1846:326-41. doi: 10.1016/j. bbcan.2014.05.004.

53. Dettmer K, Nürnberger $N$, Kaspar $H$, Gruber MA, Almstetter MF, Oefner PJ. Metabolite extraction from adherently growing mammalian cells for metabolomics studies: optimization of harvesting and extraction protocols. Anal Bioanal Chem. 2011; 399:1127-39. doi: 10.1007/ s00216-010-4425-x.

54. Gyori BM, Venkatachalam G, Thiagarajan PS, Hsu D, Clement M-V. OpenComet: an automated tool for comet assay image analysis. Redox Biol. 2014; 2:457-65. doi: 10.1016/j.redox.2013.12.020.

55. Blanco VM, Chu Z, Vallabhapurapu SD, Sulaiman MK, Kendler A, Rixe O, Warnick RE, Franco RS, Qi X, Blanco VM, Chu Z, Vallabhapurapu SD, Sulaiman MK, et al. Phosphatidylserine-selective targeting and anticancer effects of SapC-DOPS nanovesicles on brain tumors. Oncotarget. 2014; 5:7105-18. doi: 10.18632/oncotarget.2214. 